\title{
Annealing of ion-irradiated hexagonal boron nitride on $\operatorname{Ir}(111)$
}

\author{
Philipp Valerius, ${ }^{1}$ Charlotte Herbig, ${ }^{1,}{ }^{*}$ Moritz Will, ${ }^{1}$ Mohammad A. Arman, ${ }^{2}$ Jan Knudsen, ${ }^{3}$ Vasile Caciuc, ${ }^{4}$ \\ Nicolae Atodiresei, ${ }^{4}$ and Thomas Michely ${ }^{1}$ \\ ${ }^{1}$ II. Physikalisches Institut, Universität zu Köln, Zülpicher Straße 77, 50937 Köln, Germany \\ ${ }^{2}$ Division of Synchrotron Radiation Research, Lund University, Box 118, 22100 Lund, Sweden \\ ${ }^{3}$ MAX IV Laboratory and Division of Synchrotron Radiation Research, Lund University, Box 118, 22100 Lund, Sweden \\ ${ }^{4}$ Forschungszentrum Jülich, Wilhelm-Johnen-Straße, 52428 Jülich, Germany
}

(Received 11 September 2017; published 8 December 2017)

\begin{abstract}
Annealing of a monolayer of hexagonal boron nitride destroyed by Xe ion irradiation gives rise to rich structural phenomena investigated here through a combination of scanning tunneling microscopy, low-energy electron diffraction, x-ray photoelectron spectroscopy, and density functional theory calculations. We find selective pinning of vacancy clusters at a single specific location within the moiré formed by hexagonal boron nitride (h-BN) and the Ir substrate, crystalline Xe at room temperature of monolayer and bilayer thickness sealed inside h-BN blisters, standalone blisters only bound to the metal at temperatures where boron nitride on $\operatorname{Ir}(111)$ decomposes, and finally a pronounced threefold symmetry of all morphological features due to the preferential formation of boron-terminated zigzag edges that firmly bind to the substrate. The investigations give clear insight into the relevance of the substrate for the damage creation and annealing in a two-dimensional layer material.
\end{abstract}

DOI: 10.1103/PhysRevB.96.235410

\section{INTRODUCTION}

A monolayer of hexagonal boron nitride (h-BN) is isoelectronic and isostructural to graphene ( $\mathrm{Gr}$ ). It is distinct from $\mathrm{Gr}$ as its basis is formed by two inequivalent atoms, a boron and a nitrogen atom, in place of two equivalent $\mathrm{C}$ atoms as for $\mathrm{Gr}$. This gives rise to a fundamental difference in the electronic properties: While $\mathrm{Gr}$ is a zero-band-gap semiconductor, h-BN is an excellent insulator with a bandgap of about $5 \mathrm{eV}$ [1]. Because of its inertness, flatness, structural perfection, and insulating character, cleaved bulk hexagonal boron nitride or monolayers of h-BN are now widely used as substrates for organic molecules [2,3], topological insulators [4], and graphene [5]. Hexagonal boron nitride is established as a key component in vertical van der Waals heterostructures [6-8]. Its high mechanical strength, low friction, excellent heat conductivity, resistance against degradation at high temperatures, and the ability to produce it as nanosheets or wires [9] moves its application for water cleaning [10], gas separation [11], and as a component in high-strength composite materials [12] into reach. Besides exfoliation, chemical vapor deposition on catalytically active metals is a way to prepare well-defined monolayers [13]. Examples are epitaxial h-BN layers on $\mathrm{Rh}(111)$ [14-16], $\operatorname{Ru}(0001)$ [17-19], $\operatorname{Pt}(111)$ [20], and $\operatorname{Ir}(111)$ [21-27].

The nonequivalence of the two basis atoms in $\mathrm{h}$-BN forming the $\mathrm{sp}^{2}$ hybridized honeycomb causes not only a qualitative difference in the electronic structure to Gr but has also a profound effect on damage formation and annealing. Gr displays high flexibility in bond reorganization while h-BN does not. For free-standing Gr, many defect structures involving 5- and 7-membered $\mathrm{C}$ rings have been observed in electron irradiation studies using a transmission electron microscope (TEM) [28-32]. Even single vacancies reconstruct into a 5-9 defect (a paired configuration of a 5- and a 9-membered $\mathrm{C}$ ring), leaving behind only one dangling bond [33,34].

\footnotetext{
*Present address: Department of Physics, University of California at Berkeley, USA.
}

Continued electron irradiation of Gr transforms the honeycomb lattice into two-dimensional (2D) amorphous carbon, still $\mathrm{sp}^{2}$ bonded, but consisting of a random arrangement of polygons, predominantly 5-, 6-, and 7-membered C rings [29]. In h-BN, formation of 5- and 7- membered rings would involve homoelemental bonds which are substantially weaker and thus unfavorable. Therefore, single vacancies in free-standing h-BN are unreconstructed with three dangling bonds. Continued electron irradiation of $\mathrm{h}-\mathrm{BN}$ causes only the ejection of atoms and subsequent formation of triangular, $\mathrm{N}$-terminated holes in the h-BN honeycomb, but no bond reorganization $[28,35]$. The differences caused by the homo- versus heteroelemental bonding are not limited to the structure of the irradiation damage itself. Based on density functional theory (DFT) calculations, damage annealing in h-BN must be assumed to be substantially impaired. For example, the monovacancy migration energy in free-standing Gr is about $1.4 \mathrm{eV}$ [36], while in h-BN it is $2.6 \mathrm{eV}$ for a $\mathrm{B}$ and $5.8 \mathrm{eV}$ for an $\mathrm{N}$ vacancy [37].

In the past few years, ion-induced damage and annealing of $\mathrm{Gr}$ on a metal substrate has been intensely investigated: trapping of single noble gas ions under $\mathrm{Gr}$ on $\mathrm{Ru}(0001)$ [38]; formation of highly pressurized gas blisters under $\mathrm{Gr}$ on $\operatorname{Pt}(111)$ [39], $\operatorname{Ir}(111)$ [40-42], $\operatorname{Ir}(100)$ [43], and $\mathrm{Ni}(111)$ $[44,45]$ upon annealing subsequent to noble gas implantation, damage evolution with ion energy [46]; formation of a regular arrangement of vacancy clusters with moiré periodicity (nanomesh) [47]; interface channeling [47]; and sputter protection of the substrate through a Gr cover [48]. In these studies, room-temperature irradiated $\mathrm{Gr}$ demonstrated a remarkable ability to self-repair upon annealing, which one might relate to the flexible bond reorganization in this material. Far less work has been conducted for h-BN on a metal substrate and a conclusive picture of ion-induced damage and annealing is missing yet. Only irradiation at very low energies, just above the displacement threshold and at extremely low fluences, has been conducted for h-BN on $\mathrm{Rh}(111)$ [49-52]. These studies found stable trapping of single Ar atoms underneath the h-BN cover and investigated their behavior upon annealing. 
To date, a one-to-one comparison of irradiation and annealing of supported h-BN versus Gr, with parameters kept identical, is missing. It would provide insight into whether the large difference found in radiation damage of free-standing h-BN and Gr through TEM studies is also reflected in the radiation damage and annealing of metal supported h-BN and Gr. Therefore, insight into the generality of the observed phenomena and the relevance of the substrate in damage formation and annealing within a 2D layer material would evolve. Here, we present a study that contributes to this goal. We conduct ion irradiation of an epitaxial h-BN monolayer on $\operatorname{Ir}(111)$ under the same conditions as used for previous work on ion irradiation of Gr on $\operatorname{Ir}(111)$ (see Refs. [42,53]).

Here, similarities in the damage evolution of h-BN and Gr on $\operatorname{Ir}(111)$ are found which enable us to uncover the decisive importance of the substrate and its interaction with the 2D layer for recovery. However, also striking differences in the reorganization of the $2 \mathrm{D}$ materials become apparent: h-BN on $\operatorname{Ir}(111)$ recovers in triangular shapes, a fact that is linked to the two nonequivalent atoms in the unit cell of h-BN. Additional remarkable observations are the direct imaging of crystalline Xe in blisters and the finding that tiny vacancy clusters in h-BN on $\operatorname{Ir}(111)$ are pinned up to $1300 \mathrm{~K}$ to a specific location in the moiré of h-BN with its substrate.

\section{METHODS}

The experiments were performed in an ultrahigh vacuum variable-temperature scanning tunneling microscopy (STM) system. The $\operatorname{Ir}(111)$ crystals were cleaned by cycles of $\mathrm{Xe}^{+}$ sputtering at room temperature or $970 \mathrm{~K}$ and flash annealing at $1570 \mathrm{~K}$. A closed monolayer of h-BN was grown by exposing $\operatorname{Ir}(111)$ to a dose of $100 \mathrm{~L}$ of borazine at $1250 \mathrm{~K}$. The ion-irradiation experiments were performed at $300 \mathrm{~K}$. The ion flux was adjusted through a Faraday cup moved to the sample position prior to each irradiation experiment. The ion fluence was set by the irradiation time at a given ion flux and is given in monolayer equivalents (MLE), where 1 MLE corresponds to the surface atomic density of $\operatorname{Ir}(111)$, i.e., $1.57 \times 10^{19}$ particles per $\mathrm{m}^{2}$. $\mathrm{Xe}^{+}$ion energies of 0.5 and $3 \mathrm{keV}$ were used. Annealing intervals were $300 \mathrm{~s}$ at $1000 \mathrm{~K}$ and $120 \mathrm{~s}$ at $1300 \mathrm{~K}$, and flash annealing at $1500 \mathrm{~K}$ as well as $1550 \mathrm{~K}$.

STM imaging was conducted at room temperature. The sample bias $U_{\mathrm{s}}$ and the tunneling current $I_{\mathrm{t}}$ are specified for each topograph in the corresponding caption. Low-energy electron diffraction (LEED) patterns were digitally recorded over an energy range from about 20 to $200 \mathrm{eV}$.

The x-ray photoelectron spectroscopy (XPS) measurements were performed at beamline I311, MAX-lab, Lund University [54]. The B $1 s$ and $\mathrm{N} 1 s$ core levels were probed with photon energies of 284 and $500 \mathrm{eV}$ and total energy resolutions of 72 and $155 \mathrm{meV}$, respectively. The XPS data were processed with a polynomial background subtraction of fifth order and fitted with Doniach-Sunjic line shapes using the LevenbergMarquardt minimization algorithm. The fitting parameters are listed in Table S1 of the Supplemental Material [53]. Binding energies were calibrated to the Fermi edge.

Based on DFT [55], our ab initio calculations have been performed by employing the projector augmented wave method [56] to describe electron-ion interactions as implemented in the
VASP code [57-59]. The projector augmented wave method (PAW) pseudopotentials used in this study were generated with the Perdew-Burke-Ernzerhof (PBE) exchange-correlation energy functional [60] while the cutoff energy that determines the size of the plane-wave basis was set to $500 \mathrm{eV}$. Additionally, the van der Waals (vdW) interactions that bind the h-BN islands to the $\operatorname{Ir}(111)$ surface were taken into account using the nonlocal correlation energy functional vdW-DF2 [61] together with the exchange energy functional developed by Hamada [62]. Furthermore, the relaxed geometries of the h-BN islands/Ir(111) configurations considered in this study were obtained when the calculated forces were smaller than a threshold value of $10 \mathrm{meV} / \AA$. The adsorption energies of the h-BN nanoflakes are the system energy minus the sum of the energies of the $\operatorname{Ir}(111)$ slab, the single Xe atoms, and the free-standing h-BN nanoflake. Three layers of Ir and a vacuum height of $10 \AA$ were used in the calculations.

\section{RESULTS}

\section{Scanning tunneling microscopy and low energy electron diffraction experiments}

After entirely destroying h-BN on $\operatorname{Ir}(111)$ through ion irradiation, the material can be re-established by annealing at elevated temperatures. An overview on these processes is provided by the series of scanning tunneling microscopy (STM) topographs in Figs. 1(b)-1(f).

\section{The structure of h-BN/Ir(111)}

For reference, Fig. 1(a) displays the morphology of pristine h-BN after growth. Because of the mismatch of the lattice parameters of h-BN and the $\operatorname{Ir}(111)$ surface lattice, a moiré emerges. It has a periodicity of $2.91 \mathrm{~nm}$ [26] and can be recognized in Fig. 1(a) through the periodicity of the dark valleys, one valley located in the center of each unit cell. At the valley location, h-BN on $\operatorname{Ir}(111)$ is chemisorbed, with chemical bonds between $\mathrm{N}$ atoms atop of $\mathrm{Ir}$ atoms and $\mathrm{B}$ atoms residing in threefold-coordinated Ir hollow sites, either regular face centered cubic (fcc) or faulted hexagonal close packed (hcp) ones. Otherwise, h-BN is physisorbed on Ir. The two lattices are aligned with their dense-packed rows to good approximations. On average, $(11.7 \times 11.7) \mathrm{h}-\mathrm{BN}$ unit cells rest on $(10.7 \times 10.7) \operatorname{Ir}(111)$ unit cells [26]. In the chemisorbed valley region, the separation of the h-BN layer and the $\operatorname{Ir}(111)$ substrate is only about $2.2 \AA$, while in the physisorbed areas it is about $3.7 \AA$ [26]. The resulting corrugation of $1.5 \AA$ is much larger as the apparent corrugation measured by STM: The line profile in Fig. 1(a) depicts an apparent corrugation of at most $0.5 \AA$. For the insulator h-BN, the STM contrast and corrugation vary strongly with the tunneling parameters, e.g., the valleys may even appear as protrusions $[25,63]$.

\section{Morphology of h-BN/Ir(111) after ion irradiation}

After h-BN growth, the sample shown in Fig. 1(a) was exposed to $0.1 \mathrm{MLE} 3 \mathrm{keV} \mathrm{Xe}^{+}$irradiation at normal incidence and $300 \mathrm{~K}$. Figure 1(b) shows an STM topograph of the morphology directly after ion irradiation. The surface is rough, with a high density of protrusions as well as depressions on 

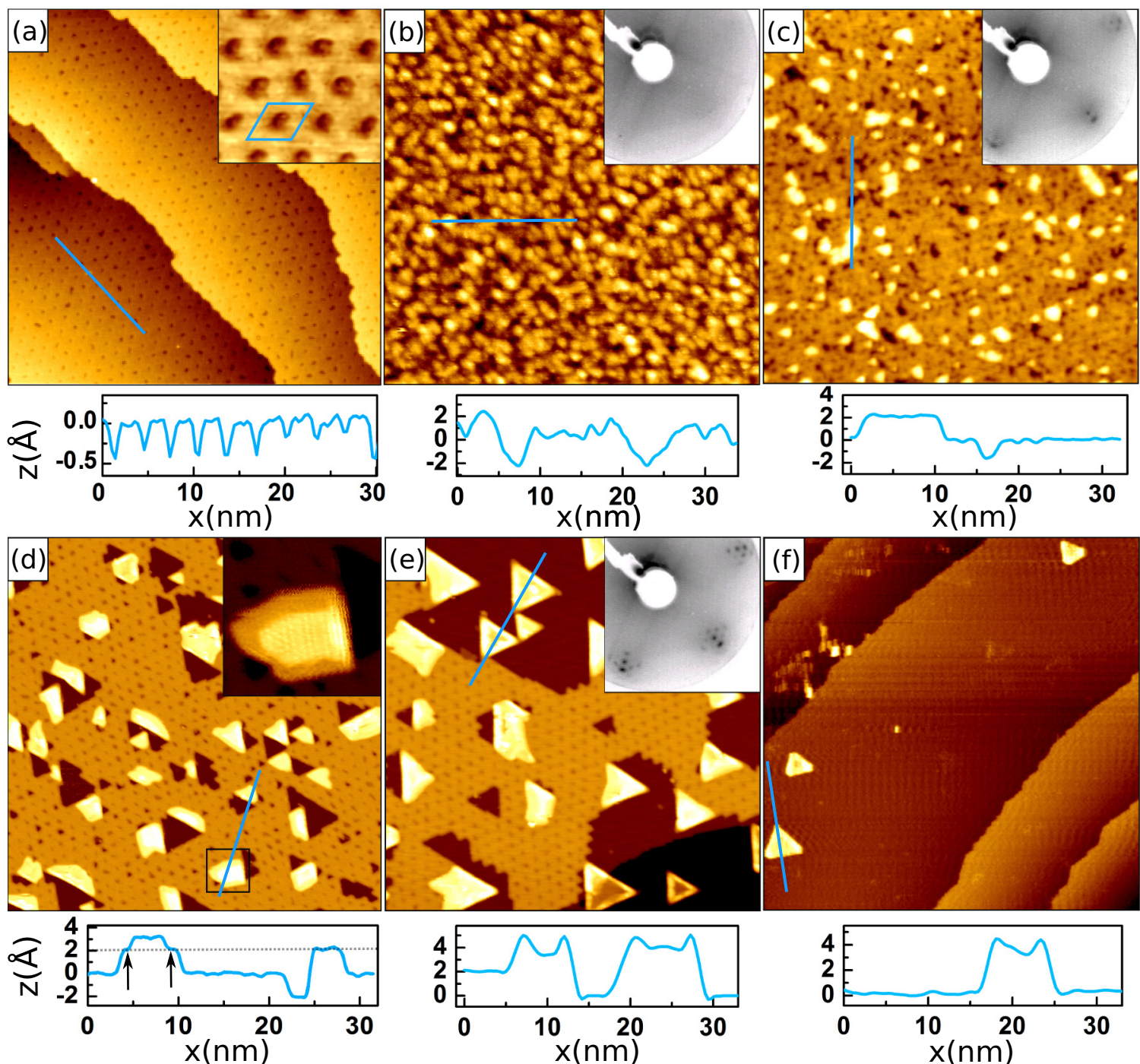

FIG. 1. STM topographs of (a) pristine h-BN/Ir(111), (b) after exposure of the sample in panel (a) to $0.1 \mathrm{MLE}^{\circ} 3 \mathrm{keV} \mathrm{Xe}^{+}$at $300 \mathrm{~K}$, and after additional annealing at (c) $1000 \mathrm{~K}$, (d) $1300 \mathrm{~K}$, (e) $1500 \mathrm{~K}$, and (f) $1550 \mathrm{~K}$. Height profiles are along the blue lines indicated in the topographs. In panels (d) and (e), the contrast is chosen to highlight the vacancy clusters and the corrugation in the h-BN layer. The inset in panel (a) is a contrast-enhanced enlargement. The rhomb indicates the moiré unit cell. The insets in panels (b), (c), and (e) are 68-eV LEED patterns taken after the corresponding annealing step. The inset in panel (d) is a contrast-enhanced enlargement of the area indicated by the black frame in panel (d) highlighting a blister with two layers of stacked Xe. Tunneling parameters are (a) $U_{\mathrm{b}}=-1.97 \mathrm{~V}, I_{\mathrm{t}}=1 \mathrm{nA}$, (b) $U_{\mathrm{b}}=-1.21 \mathrm{~V}, I_{\mathrm{t}}=0.39 \mathrm{nA}$, (c) $U_{\mathrm{b}}=-1.42 \mathrm{~V}, I_{\mathrm{t}}=0.06 \mathrm{nA}$, (d) $U_{\mathrm{b}}=-1.19 \mathrm{~V}, I_{\mathrm{t}}=0.09 \mathrm{nA}$, (e) $U_{\mathrm{b}}=-0.91 \mathrm{~V}, I_{\mathrm{t}}=0.23 \mathrm{nA}$, and (f) $U_{\mathrm{b}}=-1.33 \mathrm{~V}, I_{\mathrm{t}}=0.15 \mathrm{nA}$. Image size is always $90 \mathrm{~nm} \times 90 \mathrm{~nm}$. Size of inset in panel (a) is $12 \mathrm{~nm} \times 12 \mathrm{~nm}$ and in panel (d) is $16 \mathrm{~nm} \times$ $16 \mathrm{~nm}$.

the nanometer scale, and with a total corrugation of $4 \AA$. No sign of the moiré can be recognized. The roughness is partly due to the damage created in the Ir substrate in consequence of irradiation, e.g., Ir adatoms and surface vacancies (compare Ref. [42]). However, a large part of the corrugation must be due to Xe atoms and small assemblies thereof resting underneath $\mathrm{h}-\mathrm{BN}$. This statement is based on our x-ray photoelectron spectroscopy experiments that display a huge increase of the $\mathrm{Xe} 3 \mathrm{~d}$ signal after irradiation, only when an h-BN cover is present (for more information, see Fig. S2 of the SM [53]). Because of the substantial fluence of primary ions penetrating the h-BN sheet and the energized Ir recoil atoms hitting the h-BN sheet from below, it must be assumed that the structural integrity of the h-BN layer is severely disturbed. In fact, the
LEED pattern after 0.1 MLE $3 \mathrm{keV} \mathrm{Xe}{ }^{+}$irradiation shown as an inset in Fig. 1(b) displays almost invisible first-order diffraction spots of the $\operatorname{Ir}(111)$ substrate, but none of the h-BN layer. The h-BN layer appears amorphous in LEED.

\section{Irradiated h-BN/Ir(111) annealed to $1000 \mathrm{~K}$}

The sample shown in Fig. 1(b) was additionally annealed to $1000 \mathrm{~K}$ and the resulting morphology is represented in Fig. 1(c). A well-defined base level appears to be restored, but the moiré is, if at all, only faintly visible. A large number of protrusions emerge above the base level, of which the larger ones display a flat top with a height of about $2 \AA$ [compare height profile in Fig. 1(c)]. Also depressions with a frequent 
separation of $\approx 3 \mathrm{~nm}$ are present, a distance reminiscent of the moiré. The larger ones possess a depth of about $2 \AA$. We interpret the morphology as follows: A flat $\operatorname{Ir}(111)$ surface is restored under the h-BN layer after annealing at $1000 \mathrm{~K}$, since Ir adatoms and clusters thereof are mobile well below $1000 \mathrm{~K}$ and thus able to recombine with surface vacancies [47]. The depressions are interpreted as vacancy clusters (small, size less than $2 \mathrm{~nm}$ ) and vacancy islands (large, size greater than $2 \mathrm{~nm}$ ) within the h-BN layer. The vacancy structures emerge, since nitrogen and boron were sputtered during irradiation. As the vacancy islands result from aggregation, their separation and small lateral expansion implies a limited mobility of irradiation-induced vacancies at $1000 \mathrm{~K}$ on a length scale of $2 \mathrm{~nm}$. The protrusions are interpreted as Xe-filled blisters at the interface of the h-BN layer and the $\operatorname{Ir}(111)$ substrate. The well-defined apparent height of the large blisters indicates a monolayer thickness of the encapsulated Xe. The Xe blisters aggregate from smaller $\mathrm{Xe}$ entities present at $300 \mathrm{~K}$, since larger blisters minimize strain and delamination energies in the h-BN [42,43,51]. The low-energy electron diffraction (LEED) pattern shown as an inset in Fig. 1(c) displays again first-order Ir and h-BN reflexes, indicating the restoration of the substrate and h-BN layer. However, the moiré reflexes are hardly visible, assuming a poor long-range order of the h-BN layer, in agreement with the STM topographies.

\section{Irradiated h-BN/Ir(111) annealed to $1300 \mathrm{~K}$}

The next annealing step takes the sample to $1300 \mathrm{~K}$ and the corresponding morphology is shown in Fig. 1(d). The STM topograph displays a well-ordered moiré pattern, from which it is evident that the h-BN lattice structure is restored to perfection. The blisters ripened further, as obvious from their number decrease and size increase, with their lateral dimension now being in the order of $5 \mathrm{~nm}$. The blisters tend to display triangular shapes, specifically when adjacent to a vacancy island. Most of the blisters display a two-level structure with apparent heights of 2 and $3.5 \AA$, as shown by the inset of Fig. 1(d), which represents an enlargement of the blister in the black box (contrast adjusted to highlight the twolevel structure), as well as the corresponding height profile. Below we will provide evidence that the two-level appearance of the blisters is linked to a two-layer structure of the encapsulated Xe.

The vacancy aggregates display a bimodal distribution: Large aggregates - vacancy islands-extend over the area of several moiré unit cells and possess a tendency for triangular shapes. Small aggregates-vacancy clusters-are pinned to the moiré at the location of the valleys. Vacancy clusters and valleys are not always easy to distinguish, as for smaller vacancy clusters the blunt STM tip does not reach down to the substrate, resulting in a variety of depths between $\approx 2 \AA$ and $0.5 \AA$. Nevertheless, the situation is unambiguous for larger vacancy clusters and a close inspection reveals that at about $25 \%$ of the valley locations vacancy clusters are present.

The pinning of vacancy clusters precisely at the location of the valley and their large number are remarkable findings. We interpret them as follows: The configuration of a vacancy cluster at the valley location must be a a deep energetic minimum for the lateral position of the vacancy cluster. If not, the energy of a vacancy cluster at a different location would not differ much, and consequently it would be mobile at $1300 \mathrm{~K}$ and coalesce with others. Note that assuming a standard pre-exponential factor, a temperature of $1300 \mathrm{~K}$ is sufficient to overcome activation energies of more than $3 \mathrm{eV}$ on a time scale of seconds. So why does the valley location define such a deep energetic minimum for a vacancy cluster? By removing the core of the valley, the edge atoms of the resulting vacancy cluster still bind strongly to the substrate, as they are chemisorbed to $\operatorname{Ir}(111)$. Removing a similarly sized vacancy cluster outside the valley region implies weaker edge atom bonds to the substrate, as their original height was about $3.7 \AA$ above Ir, instead of $2.2 \AA$ as at the valley location, making it energetically costly to bend down to the substrate to form bonds, if possible at all. Thus, due to the inferior edge atom bonding to the substrate, vacancy clusters outside the valleys are strongly disfavored. Since the edge atom binding energy to the substrate in a favorable geometry is of the order of $2 \mathrm{eV}$ per atom [26], the enormous stability of the vacancy clusters becomes understandable. We note that a similar pinning of vacancy clusters to specific moiré sites was also found for graphene on $\operatorname{Ir}(111)[40,47]$. However, for graphene on $\operatorname{Ir}(111)$, the vacancy clusters are thermally less stable (only up to $1000 \mathrm{~K}$ ) and the location within the moiré unit cell is not uniquely defined. In fact, the graphene moiré unit cell on $\operatorname{Ir}(111)$ has two distinct preferential locations for a vacancy cluster, which are both populated.

Image analysis of topographs after annealing at $1300 \mathrm{~K}$, like the one shown in Fig. 1(d), gives on average for the removed h-BN layer an area fraction of $13.8 \%$. Assuming this loss to result from sputtering, a joint boron and nitrogen sputtering yield of $Y_{\mathrm{h}-\mathrm{BN}, \mathrm{Ir}}=3.3$ atoms/ion is obtained. This yield is much larger than the yield for $3 \mathrm{keV} \mathrm{Xe}$ of free-standing h-BN. It amounts to $Y_{\mathrm{h}-\mathrm{BN} \text {,free }}=1.2$ atoms/ion as estimated from the molecular dynamics data of Lehtinen et al. [64]. As there is no sign of h-BN decomposition after annealing at $1300 \mathrm{~K}$, we consider this value-though certainly subject to substantial errors - as a realistic estimate for the yield. Thus, $Y_{\mathrm{h}-\mathrm{BN}, \mathrm{Ir}}$ being almost three times larger than $Y_{\mathrm{h} \text {-BN,free }}$ is a remarkable quantitative difference. We attribute this difference to the additional sputtering of h-BN caused by recoiling $\mathrm{Ir}$ atoms created in the collision cascade in the substrate and to recoiling primary ions, as it was found for $\operatorname{Gr} / \operatorname{Ir}(111)$ [65].

\section{Irradiated h-BN/Ir(111) annealed to $1500 \mathrm{~K}$}

The next annealing step is at $1500 \mathrm{~K}$ and the resulting morphology is presented in Fig. 1(e). The most remarkable change is the absence of vacancy clusters pinned to the moiré. Evidently, vacancy clusters dissolved and ripened to large vacancy islands. The moiré does not anymore represent an obstacle for vacancy diffusion. The area of vacancy islands is about twice as large as the summed area of vacancy islands and clusters at $1300 \mathrm{~K}$. This is a first indication for the partial decomposition of the h-BN layer at $1500 \mathrm{~K}$.

Blisters ripened also in this annealing step, with a decrease in number and an increase in size. The remaining blisters are mostly triangular in shape. Blisters not bound to the rest of the h-BN layer and bound with their edges only to the Ir(111) substrate (standalone blisters) are present within large 
vacancy islands [compare blisters in the vacancy island in the upper part of Fig. 1(e)]. As the height profile in Fig. 1(e) indicates, these blisters are often not uniformly curved outward (negative curvature), but display a depression in their center. This issue will be discussed below in more detail. Remarkably, in the lower right part of Fig. 1(e), a blister extends over an Ir substrate step. It highlights the flexibility of the blister, which enables efficient trapping of Xe underneath, even when its baseline is not bound to an atomically flat plane.

The inset of Figure 1(e) displays the LEED pattern after annealing to $1500 \mathrm{~K}$ (indistinguishable from the $1300 \mathrm{~K}$ pattern, which is not shown). At least two orders of moiré reflexes are visible, indicating the perfect long-range order of the h-BN layer. The quality of the LEED patterns is comparable to as grown h-BN on $\operatorname{Ir}(111)$ (compare Ref. [26]). This implies the complete recovery of the h-BN lattice and the absence of defects on the length scale of the order of the LEED transfer width of around $10 \mathrm{~nm}$, in perfect agreement with the STM topographs.

\section{Irradiated h-BN/Ir(111) annealed to $1550 \mathrm{~K}$}

The final annealing step of the $3 \mathrm{keV} \mathrm{Xe}^{+}$irradiated $\mathrm{h}-\mathrm{BN} / \mathrm{Ir}(111)$ sample is at $1550 \mathrm{~K}$. The STM topograph of Fig. 1(f) makes plain that the h-BN layer decomposed almost completely during this annealing step, such that bare Ir terraces are present nearly in the entire topograph. Just a few small standalone blisters have survived this thermal treatment. A possible explanation for this finding is that as h-BN on $\mathrm{Ir}$ decomposes first in the valleys [26], this decay channel is blocked by the Xe filling of the blisters. Direct contact between the h-BN and the Ir substrate is only present at the edges. The Xe filling will strain these edge bonds and make close approach of the B or $\mathrm{N}$ edge atoms to the Ir substrate atoms more difficult, thereby diminishing the decay rate. The fact that after annealing at $1550 \mathrm{~K}$ the blisters are much smaller than at $1500 \mathrm{~K}$ indicates that they are already the result of a shrinking process, presumably due to the loss of h-BN from the blister edges.

Irradiation damage and annealing morphology of the h$\mathrm{BN}$ layer-including the phenomena of amorphization, blister formation, vacancy cluster trapping, emergence of standalone blisters, and the evolution of triangular shapes - are obtained also for other ion energies and depend only quantitatively on ion energy, fluence, and species. An example for a 500-eV Xe ${ }^{+}$ irradiation sequence is provided as Fig. S1 of the SM [53].

\section{A. Decomposition of $\mathbf{h}-\mathrm{BN}$ and blister effects after annealing irradiated $\mathrm{h}-\mathrm{BN} / \mathrm{Ir}(111)$ at $1500 \mathrm{~K}$}

After annealing irradiated h-BN/Ir(111) to $1500 \mathrm{~K}$, we noticed above that there are indications for the onset of h-BN decomposition (reduced h-BN coverage). Also blisters with a depression in their interior were observed. We will elaborate on these findings using the information provided in Fig. 2.

Figure 2(a) displays an STM topograph after annealing at $1500 \mathrm{~K}$ with an $\operatorname{Ir}(111)$ substrate step crossing from left to right in an area where the h-BN layer is largely removed. Straight step segments are visible (highlighted by white arrows), which are decorated by a line approximately $0.5 \AA$ higher than the upper terrace [compare line profile in Fig. 2(b)]. A higher

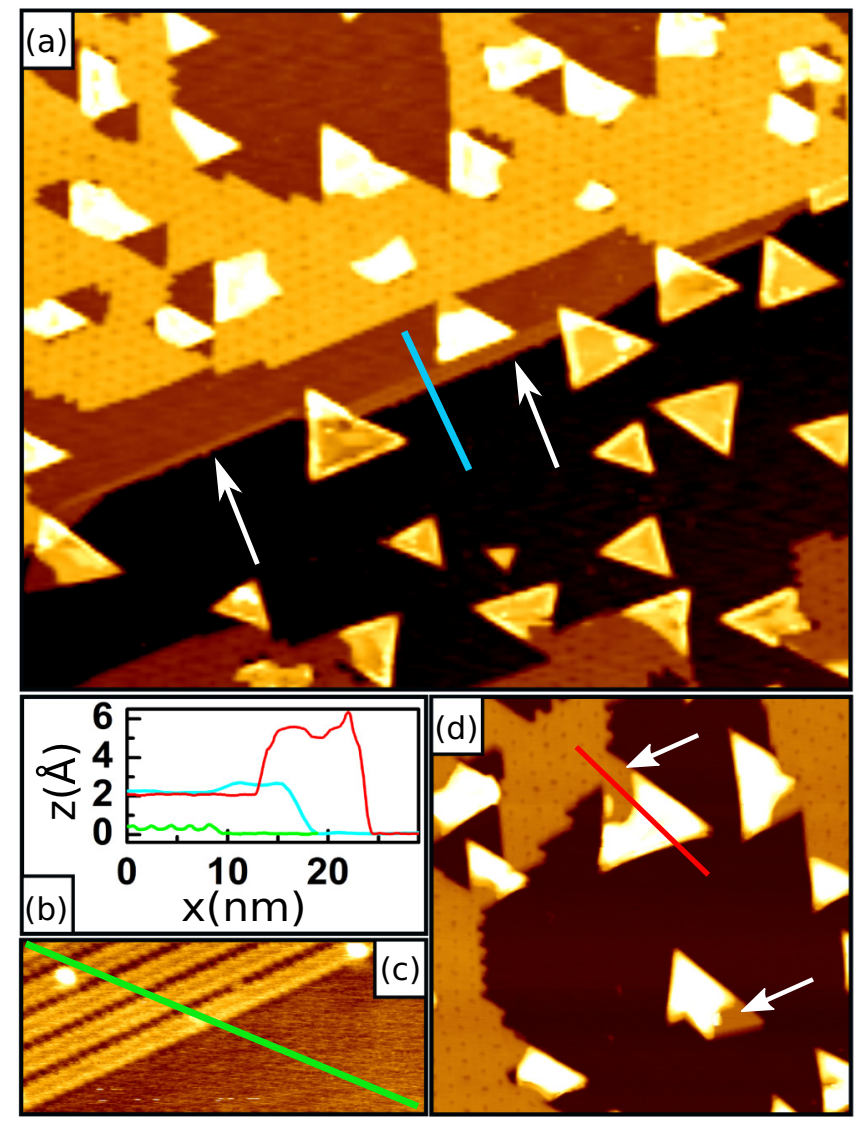

FIG. 2. (a) STM topograph after $0.1 \mathrm{MLE} 3 \mathrm{keV} \mathrm{Xe} \mathrm{Xe}^{+}$irradiation of $\mathrm{h}-\mathrm{BN} / \operatorname{Ir}(111)$ and additional annealing at $1500 \mathrm{~K}$. Contrast is adjusted to highlight the $\mathrm{B}$ structures at the Ir step edge, also indicated by white arrows. (b) Height profiles along blue line in panel (a), green line in panel (c), and red line in panel (d). (c) Higher resolution STM topograph of a bundle of stripes after annealing the sample to 1550 K. (d) STM topograph after preparation as in panel (a) with partly relaminated triangular blisters (highlighted by white arrows). Contrast is adjusted to highlight relaminated parts of the blisters. See text. Tunneling parameters are (a) $U_{\mathrm{b}}=-1 \mathrm{~V}, I_{\mathrm{t}}=0.06 \mathrm{nA}$, (c) $U_{\mathrm{b}}=-0.5 \mathrm{~V}, I_{\mathrm{t}}=0.57 \mathrm{nA}$, (d) $U_{\mathrm{b}}=-1.5 \mathrm{~V}, I_{\mathrm{t}}=0.04 \mathrm{nA}$. Image sizes are (a) $150 \mathrm{~nm} \times 124 \mathrm{~nm}$, (c) $18 \mathrm{~nm} \times 8 \mathrm{~nm}$, and (d) $70 \mathrm{~nm} \times$ $70 \mathrm{~nm}$.

resolution image of a bundle of such lines is provided in Fig. 2(c), where a height variation of $0.5 \AA$ is also apparent for each stripe with respect to the Ir substrate [see green height profile along green line in Fig. 2(c)]. Such features were observed neither on the clean $\operatorname{Ir}(111)$ sample nor after annealing at $1300 \mathrm{~K}$ or below. The stripes are interpreted as B ribbons formed on the $\operatorname{Ir}(111)$ surface. Based on their real space periodicities, the ribbons are identified with the $(6 \times 2)$ structure found by Petrovic et al. as a consequence of h-BN decomposition on $\operatorname{Ir}(111)$ [27] after growth at $1370 \mathrm{~K}$. $\mathrm{H}-\mathrm{BN}$ decomposition on $\operatorname{Ir}(111)$ at the same temperature and the presence of a decomposition-related B $1 s$ XPS component was already noticed by Usachov et al. [24]. According to the data of Petrović et al. [27], the ribbons organize upon cooling at around $1220 \mathrm{~K}$, either through condensation of B adatoms or segregation of $\mathrm{B}$ that had been dissolved in the selvage after h-BN decomposition. The boron sheets realized by Feng 
et al. [66] through boron molecular beam epitaxy on $\operatorname{Ag}(111)$ display a similar striped appearance as the ribbons observed here, which may be a hint for a similarity in structure. We note that nitrogen stemming from the decomposition of h-BN will rapidly desorb as $\mathrm{N}_{2}[67,68]$.

Figure 2(d) highlights a few blisters after annealing at $1500 \mathrm{~K}$. White arrows indicate two triangular blisters, which are partly laminated to the Ir substrate and even display the moiré pattern. We assume that at $1500 \mathrm{~K}$ the blisters are fully developed triangles and are purely curved outward (only negative curvature). Their curvature and volume at $1500 \mathrm{~K}$ is determined by the quasiequilibrium among pressure-volume $(\mathrm{pV})$ work (to be done upon compression), strain energy (gained upon compression), and adhesion of h-BN to $\operatorname{Ir}(111)$ (gained upon relamination associated with compression). Upon cooling, the pressure in the blisters lowers-it would drop by a factor of 5 for an ideal gas-and the energy balance favors relamination to the substrate, until the gain of adhesion energy is again balanced by $\mathrm{pV}$ work done. This results in partly relaminated blisters, leaving the characteristic triangular blister shape still visible. In Fig. 1(e) and the associated height profile [see also red curve of 2(b)], we also noticed that some blisters display a depression in their center. We assume that also the blisters with depressions in their center result from the pressure drop upon cooling and are an intermediate stage prior to relamination.

\section{B. Xe nanocrystals in blisters after ion irradiation}

For blisters formed as a consequence of noble gas irradiation of Gr, it has been proposed several times that these blisters are pressurized [42-45]. In fact, Zamborlini et al. [43] and Larciprete et al. [45] even proposed solid Ar in Gr blisters on a metal substrate, when at room temperature. This inference was based on estimates of the pressure inside the blister. Here we will provide direct evidence for the solid state of $\mathrm{Xe}$ in blisters at room temperature.

Figure 3 presents two high-resolution STM topographs, where solid Xe is imaged through the h-BN layer. Such atomic resolution imaging through a 2 D-layer cover is by now a standard technique to obtain information on the substrate and intercalation layers [69-72]. Figure 3(a) displays flat blisters after $0.5 \mathrm{keV}$ irradiation and subsequent annealing at $1000 \mathrm{~K}$. Their height is $\approx 2 \AA$ (see associated height profile) and the contrast is tuned to the top level of the blisters (the morphology is presented as inset). An additional hexagonal superstructure aligned to the blister edges and with an average periodicity of $(4.2 \pm 0.1) \AA$ is visible. The superstructure is not linked to the h-BN lattice, which would give a periodicity of $\approx 2.5 \AA$. It is interpreted as due to a single layer of compressed Xe on $\operatorname{Ir}(111)$. The measured nearest neighbor distance is consistent with a compressed Xe phase. Xe crystallizes as an fcc crystal with a nearest neighbor distance of $4.384 \AA$ [73] in the crystalline state below $161.7 \mathrm{~K}$ at normal pressure [74]. At $300 \mathrm{~K}$, it is solid for pressures in excess of $0.5 \mathrm{GPa}$ [75].

Figure 3(b) displays blisters with two layers of Xe, with an increasing height of about $2 \AA$ per Xe layer [compare height profile and also Fig. 1(d)]. With the contrast tuned to the top level of the blisters, again a superstructure is visible, which displays now a somewhat larger lattice of $(4.6 \pm 0.1) \AA$. This

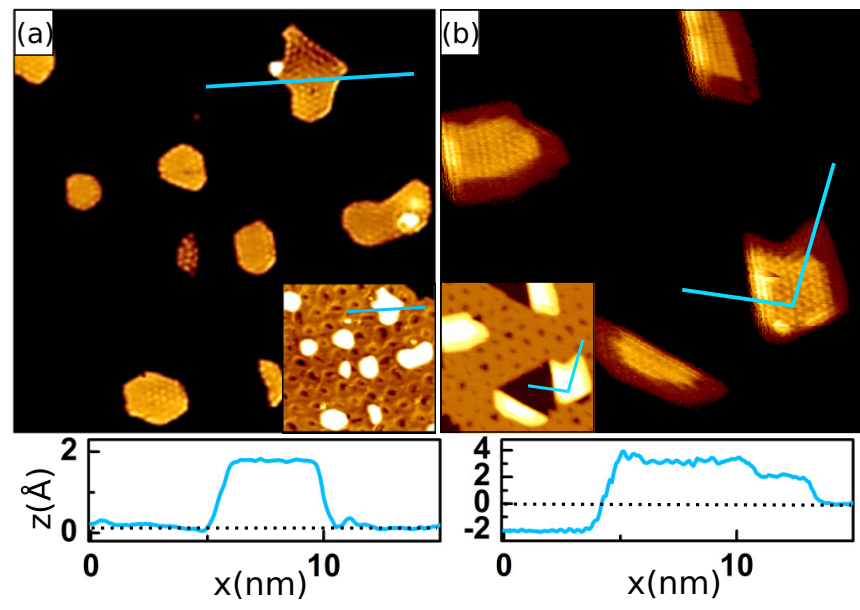

FIG. 3. (a) STM topograph $\left(U_{\mathrm{b}}=-0.23 \mathrm{~V}, I_{\mathrm{t}}=2 \mathrm{nA}\right)$ of $\mathrm{h}-$ $\mathrm{BN} / \mathrm{Ir}(111)$ after $0.5 \mathrm{keV} \mathrm{Xe}^{+}$irradiation and subsequent annealing at $1000 \mathrm{~K}$ and associated height profile along blue line (also indicated in the inset). (b) STM topograph $\left(U_{\mathrm{b}}=-1.09 \mathrm{~V}, I_{\mathrm{t}}=0.11 \mathrm{nA}\right)$ of h-BN/Ir(111) after $3 \mathrm{keV} \mathrm{Xe} \mathrm{Xe}^{+}$irradiation and subsequent annealing at $1300 \mathrm{~K}$ and associated height profile, where zero height is set to the base level of h-BN (marked with dotted line). Image size is always $27 \mathrm{~nm} \times 27 \mathrm{~nm}$. The contrast is adjusted to highlight the superstructure on top of the Xe-filled blisters. The insets display the topograph areas in the usual contrast.

lattice parameter of the two-layer Xe crystallites is consistent with the higher annealing temperature and the larger size of the aggregates, both factors which tend to lower the pressure inside the cavities.

The superstructure indicative of solid Xe was observed by us under a variety of conditions, including also ion exposure at elevated temperature and without additional annealing. Nevertheless, two conditions need to be fulfilled: The blisters possess a certain lateral size enabling organization of the enclosed $\mathrm{Xe}$ - the superstructure is not observed after roomtemperature irradiation - and the pressure inside the blisters is large enough to enable the crystalline state of Xe. As the large blisters formed after annealing to $1500 \mathrm{~K}$ possess a substantially lower pressure, according to our discussion above, it is consistent that we were not able to detect a Xe-induced superstructure in them.

\section{Shape evolution and step edges}

One of the most remarkable features in the erosion morphology of h-BN/Ir(111) is the evolution of pronounced triangular shapes, which we will attribute in the following to result from the energetic preference of a specific step edge type.

The topograph represented by Fig. 4(a) highlights the morphology after annealing at $1300 \mathrm{~K}$ on a larger scale and in more detail. A number of interesting features are observable: (i) Blisters and vacancy islands display a strong preference for triangular shapes. (ii) It is obvious that the edges of triangular blisters and vacancy islands are parallel to the densely packed [110] directions of $\operatorname{Ir}(111)$ [compare white arrow in Fig. 4(a)]. (iii) There is a vertical dark band in the middle of the topograph that is a vacancy island stripe crossing the image. 


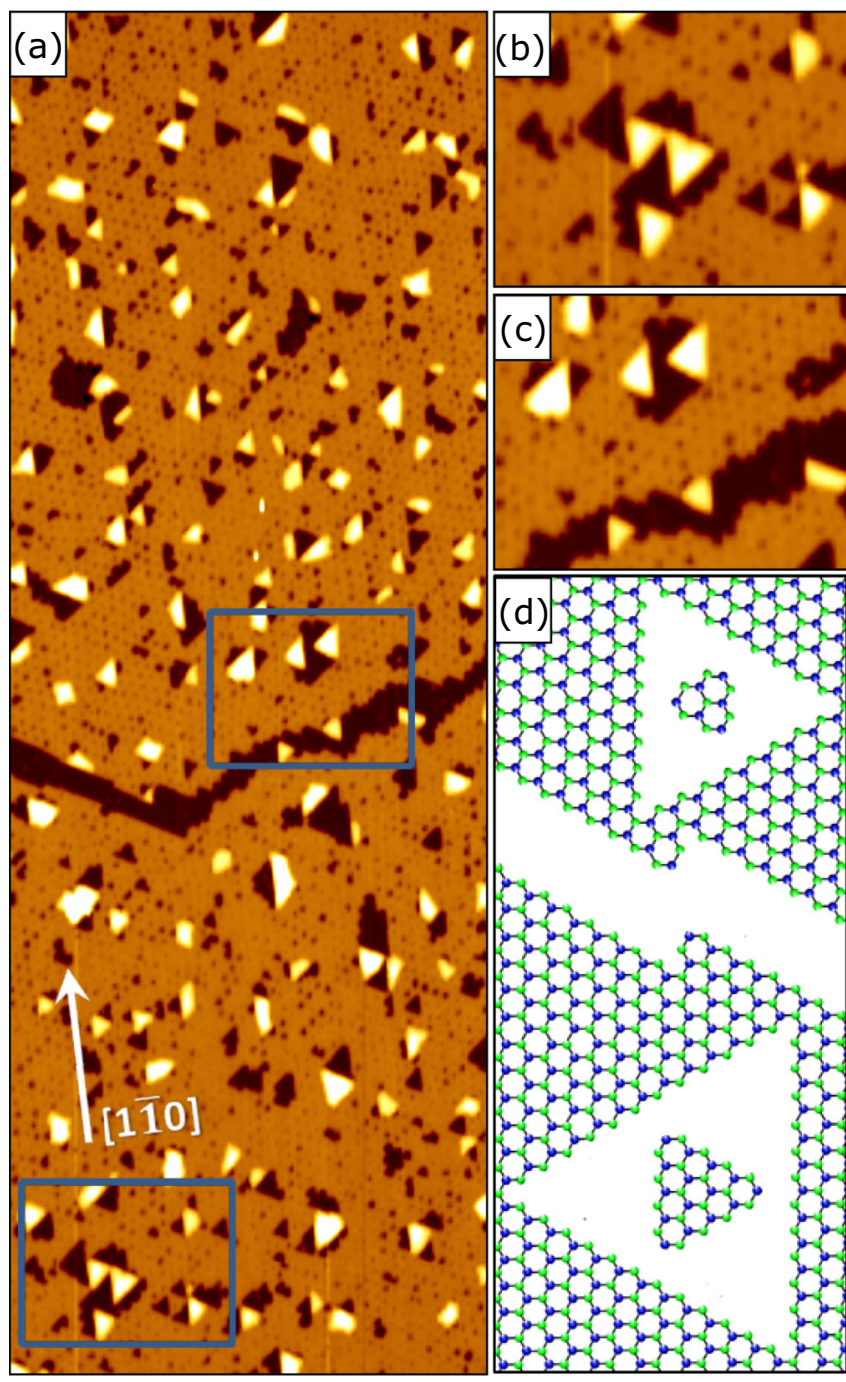

FIG. 4. (a) Large-scale STM topograph after $3 \mathrm{keV} \mathrm{Xe}{ }^{+}$bombardment and subsequent annealing at $1300 \mathrm{~K}$. Image size: $300 \mathrm{~nm}$ $\times 100 \mathrm{~nm}, U_{\mathrm{b}}=-1.19 \mathrm{~V}, I_{\mathrm{t}}=0.09 \mathrm{nA}$. Panels (b) and (c) show enlargements ( $36 \mathrm{~nm} \times 42 \mathrm{~nm}$ ) of boxes in panel (a). (d) Ball model of the h-BN layer with vacancy islands, $\mathrm{h}-\mathrm{BN}$ adatom islands, and a $180^{\circ}$ rotational domain boundary. See text.

(iv) Neighboring blisters and vacancy islands tend to have opposing triangular orientations; i.e., the triangular shapes of blisters and vacancy islands are rotated by $180^{\circ}$ with respect to each other [compare also Figs. 4(b) and 4(c)]. (v) Surprisingly, left and right of the vacancy island stripe the orientations of triangular blisters are rotated by $180^{\circ}$ with respect to each other, just as the orientations of triangular vacancy islands. (vi) The most perfect triangular blister shapes are observed for free-standing blisters surrounded by bare $\operatorname{Ir}(111)$, i.e., for blisters disconnected from the h-BN sheet and located in a large vacancy island structure. This feature is highlighted by the two enlargements in Figs. 4(b) and 4(c) [also compare Fig. 1(e)]. (vii) There is a clear tendency to find blisters next to vacancy islands and vice versa. The two defect features are apparently correlated to each other.

Since edges in the densely packed directions of h-BN (zigzag edges) are aligned with the densely packed substrate directions [26], the first two observations (i) and (ii) imply that the edges of h-BN formed during annealing have a strong preference for zigzag edges, be it a blister or vacancy island edge.

The vertical dark band separating Fig. 4(a) in two parts [observation (iii)] is interpreted as indicating the former position of a rotational domain boundary [26]. Hexagonal $\mathrm{BN}$ on a densely packed surface comes usually in two distinct rotational domains, which emerge from each other by interchanging the locations of $\mathrm{B}$ and $\mathrm{N}$ atoms (or by rotating a domain by $180^{\circ}$ ). Since their energy difference is small, they typically nucleate with close to equal probability during h-BN growth. Where two rotational domains touch, a domain boundary results, which is well recognized in STM topographs of nonirradiated h-BN on $\operatorname{Ir}(111)$ (compare also Refs. $[21,26,76])$. The internal structure of a domain boundary between two opposite domains is an intriguing problem that is not yet solved, but evidently it is a high energy defect and thus similar to an existing $\mathrm{h}-\mathrm{BN}$ island edge, a sink for migrating vacancies. As we never observed such vacancy stripes after irradiation at room temperature, we conclude that during annealing vacancies precipitate at the domain boundary and thereby form a vacancy island stripe.

Through DFT calculations, Farwick zum Hagen et al. [26] identified that the boron-terminated h-BN zigzag step edge on $\operatorname{Ir}(111)$ is preferred in energy by far compared to the $\mathrm{N}$-terminated zigzag edge. Therefore, one expects that all step edges forming the triangular structures are boron terminated. Assuming this, the triangular shapes forming blisters and vacancy islands must be rotated by $180^{\circ}$, as observed (iv). Likewise, this also implies that the shapes of blisters in the two different rotational domains are rotated by $180^{\circ}$, as told by observation (v). The same holds for the shapes of vacancy islands.

Since the triangular shapes are driven by step edge energy minimization, it is clear that blisters do not display a triangular shape when located within the h-BN sheet. The triangular shape is observed only for blisters in contact with h-BN step edges and as it is better developed, the less contact the blister has with the surrounding h-BN sheet. This explains observation (vi).

Finally, the tendency for a positional correlation of blisters and vacancy island edges [observation (vii)] is not as straightforward to understand as the other features. The explanation starts with the observation that a line profile through a blister within the h-BN plane needs to start from zero slope, curves away from its base (positive curvature), changes the sign of curvature at the protrusion top (negative curvature), and must turn to positive curvature again to match the zero slope of the h-BN plane on the other side. In contrast, when a blister is bound to an island edge, the need to merge the blister with zero slope into the h-BN layer is lifted. An h-BN edge may bind with nonzero slope to the Ir substrate. This is even likely to be energetically favorable, as for an inclined h-BN sheet less bond deformation is necessary to match the dangling $\mathrm{sp}^{2}$ edge bonds to the substrate. The need for bending with positive curvature next to the base of the protrusion is released and consequently bending energy is saved. We thus assume blisters are present preferentially at vacancy island edges, because their deformation energy is lower as 


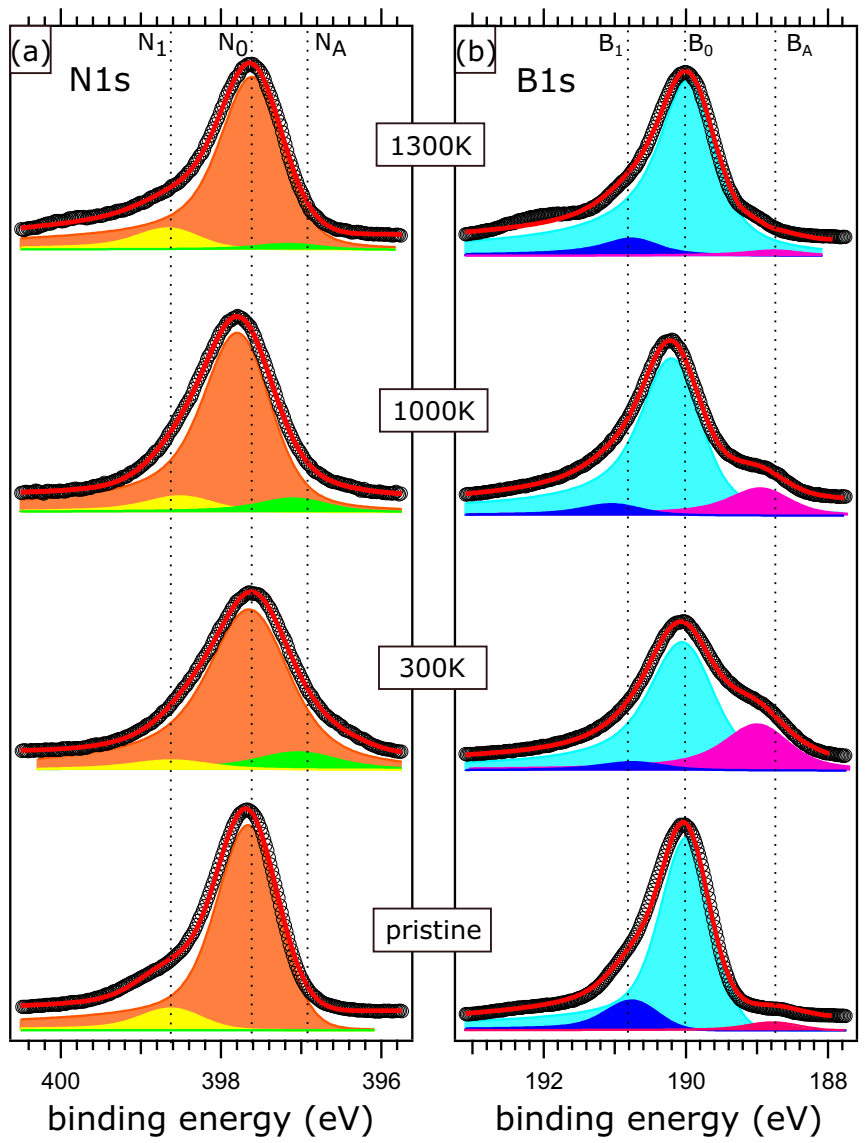

FIG. 5. XPS analysis of the (a) N $1 s$ and (b) B $1 s$ of h-BN on $\operatorname{Ir}(111)$ after the exposure to $3 \mathrm{keV} \mathrm{Xe} \mathrm{X}^{+}$ions at $300 \mathrm{~K}$ and subsequent annealing to 1000 and $1300 \mathrm{~K}$. The associated spectra of pristine $\mathrm{h}-\mathrm{BN} / \mathrm{Ir}(111)$ can be found at the bottom. Binding energies, full width at half maximum and normalized areas of the fit components are given in Table S1 of the SM [53].

compared to blisters of the same volume, but entirely within the h-BN plane.

\section{X-ray photoelectron spectroscopy}

To obtain complementary insight into the radiation damage and annealing process, we conducted XPS experiments. XP spectra of $\mathrm{N} 1 s$ and $\mathrm{B} 1 s$ core levels of pristine h-BN (lowest spectra), h-BN irradiated at $300 \mathrm{~K}$ (second lowest spectra) and h-BN annealed to 1000 and $1300 \mathrm{~K}$ (upper spectra) are displayed in Fig. 5. Consistent with literature [21,26], N $1 s$ and $\mathrm{B} 1 s$ of pristine h-BN on $\operatorname{Ir}(111)$ reveal a similar peak structure, each with a main component $\mathrm{N}_{0}$ or $\mathrm{B}_{0}$ and a small side component $\mathrm{N}_{1}$ or $\mathrm{B}_{1}$ shifted to higher binding energy by about 0.8 to $1.0 \mathrm{eV}$. The two components represent the contributions of $\mathrm{B}$ and $\mathrm{N}$ physisorbed $\left(\mathrm{N}_{0}\right.$ and $\left.\mathrm{B}_{0}\right)$ and chemisorbed in the valley region $\left(\mathrm{N}_{1}\right.$ and $\left.\mathrm{B}_{1}\right)$.

Binding energies, full width at half maximum, and normalized areas of the fit components are given in Table S1 of the SM [53]. Consistent with the literature, we allowed also asymmetric line shapes for the components [21], which in fact gave the best fit results. This relaxation appears reasonable, due to the chemisorption and substantial hybridization of the h-BN with $\operatorname{Ir}(111)$, giving rise to a non-negligible density of states of h-BN at the Fermi level $[21,26]$, and due to the multitude of slightly different chemical environments of the atoms within the groups formed by the components [77].

After irradiation at room temperature, the two-component structure of the two species $\left(\mathrm{N}_{0}\right.$ and $\mathrm{N}_{1}$ as well as $\mathrm{B}_{0}$ and $\left.\mathrm{B}_{1}\right)$ is essentially lost and the $\mathrm{N} 1 s$ and B $1 s$ peak is substantially broadened. Evidently, this implies the absence of the moiré with its distinct binding areas. Instead, a broad distribution of small chemical shifts, caused by a broad distribution of binding geometries in the heavily damaged layer, is likely. Since the main peaks are not shifted in their position with respect to the pristine case, it is not implausible to assume that the main amount of $\mathrm{B}$ and $\mathrm{N}$ atoms are still part of a disordered $\mathrm{sp}^{2}$ network.

New components $\mathrm{N}_{A}$ and $\mathrm{B}_{A}$ also emerge at lower binding energy with respect to the main peaks. Consistent with literature, we attribute this intensity to atomic species [21,24,78] detached by energetic collisions from the network, but still bound to the Ir substrate. It is obvious from the spectra in Fig. 5 that the adatom peak $\mathrm{N}_{A}$ is less pronounced compared to the adatom peak $\mathrm{B}_{A}$. While $\mathrm{B}$ atoms are firmly bound to $\mathrm{Ir}$, $\mathrm{N}$ atoms detached from the network will be able to combine to $\mathrm{N}_{2}$ and desorb [67,68], if they are not trapped underneath the damaged sheet, which is apparently the case to a significant extent.

Annealing the sample to $1000 \mathrm{~K}$ diminishes the amount of atomic species, narrows the main components $\mathrm{N}_{0}$ and $\mathrm{B}_{0}$, and implies a recovery of the chemisorption components $\mathrm{N}_{1}$ and $\mathrm{B}_{1}$. After annealing to $1300 \mathrm{~K}$, the spectra are still broadened compared to the pristine case, but the chemisorption components have further recovered and the characteristics for the atomic species $\mathrm{N}_{A}$ and $\mathrm{B}_{A}$ have disappeared. In view of the morphology of Figs. 1(d) and 4, the presence of the large number of vacancy clusters pinned to the moiré and the large vacancy islands imply a considerable number of edge atoms and near-edge atoms, which are likely to be the main cause for the residual broadening of the spectra. They could also give rise to the hump around $192 \mathrm{eV}$ in the $\mathrm{B} 1 s$ spectrum at $1300 \mathrm{~K}$ which is not accounted for in our fit by its own component.

\section{E. DFT calculations}

To gain insight into the stability and energetics of Xe-filled h-BN blisters sealed to $\operatorname{Ir}(111)$, we performed $a b$ initio calculations based on DFT. The experimentally observed triangular h-BN blisters of 5- to 10-nm edge length cannot be simulated from first principles with acceptable computational effort. Therefore, the h-BN island investigated in our simulations has an edge length of only $\approx 20 \AA$ and it is without a chemisorption valley in its interior.

The pristine triangular h-BN island binds to the iridium substrate via its zigzag edges, as experimentally observed. Our previous DFT calculations found the B termination of the zigzag edge to be energetically strongly preferred over the $\mathrm{N}$ termination [26]. The h-BN island consists of $45 \mathrm{~N}$ atoms and $52 \mathrm{~B}$ atoms, of which $3 \mathrm{~N}$ atoms and $24 \mathrm{~B}$ atoms are located at the edge. As depicted in Fig. 6(a), the inner B and $\mathrm{N}$ atoms are far away from the Ir substrate (up to $\approx 4.2 \AA$ ) and do not chemically interact with the iridium surface. On the contrary, 

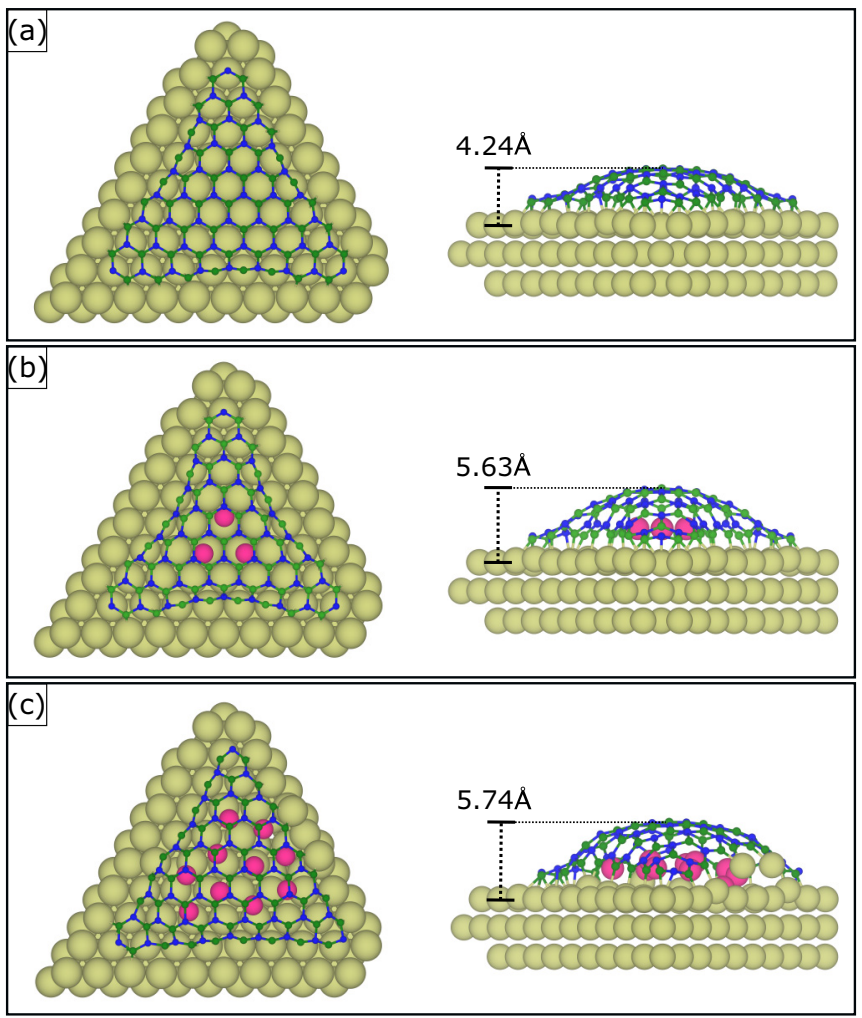

FIG. 6. Ball model representations for the relaxed DFT calculated structures of a triangular, boron-terminated h-BN island on $\operatorname{Ir}(111)$ without or with intercalated Xe. (a) h-BN island without intercalated $\mathrm{Xe}$. (b) h-BN blister consisting of an h-BN island intercalated by three $\mathrm{Xe}$ atoms. (c) h-BN blister consisting of an h-BN island intercalated by $10 \mathrm{Xe}$ atoms. Left panel: top view. Right panel: side view. Small green balls: B atoms; small, blue balls: $\mathrm{N}$ atoms; magenta balls: Xe atoms; large, light green balls: Ir atoms.

the edge atoms of the nanoisland are only $\approx 2.1 \AA$ above $\operatorname{Ir}(111)$, indicating the formation of strong $\mathrm{B}(\mathrm{N})$ - Ir chemical bonds consistent with the large calculated binding energies of $E_{\mathrm{B} \text {-edge }}=2.0 \mathrm{eV}$ and $E_{\mathrm{N} \text {-edge }}=1.6 \mathrm{eV}$ (see Ref. [26]). The large adsorption energy $E_{\mathrm{ads}}=-58.8 \mathrm{eV}$ evaluated for the pristine $\mathrm{h}-\mathrm{BN}$ island on the Ir surface is dominated by the edge bonds, but has also contributions from the energy required to deform the h-BN from its flat isolated geometry and from the van der Waals interaction of inner $\mathrm{B}$ and $\mathrm{N}$ atoms with the substrate.

In a next step, we theoretically investigated the effect of filling the h-BN island with three $\mathrm{Xe}$ atoms. The resulting blister is shown in Fig. 6(b). The Xe atoms take positions, which are $3.64 \AA$ apart and consistent with the triangular symmetry of the h-BN island. The calculated adsorption energy of $E_{\text {ads }}=-56.0 \mathrm{eV}$ indicates a slightly weaker binding as compared to the pristine case. The reduced magnitude of $E_{\text {ads }}$ is attributed to the energetic cost of additional deformation of the intercalated h-BN nanoflake as well as to the repulsive interaction between $\mathrm{Xe}$ atoms. It is important to note that the average distance between the edge atoms and Ir surface atoms is practically the same as in the pristine h-BN nanoflake. The feature underlines the strong bonds between h-BN boron edge atoms and Ir surface ones. Nevertheless, the inner B and $\mathrm{N}$
TABLE I. Calculated adsorption energies and Xe-Xe nearest neighboring distances for the h-BN nanoflakes on Ir, depending on the number of intercalated $\mathrm{Xe}$ atoms.

\begin{tabular}{lcc}
\hline \hline Xe atoms & $\mathrm{E}_{\text {ads }}[\mathrm{eV}]$ & Xe-Xe distance $[\AA]$ \\
\hline 0 & -58.8 & \\
3 & -56.0 & 3.644 \\
4 & -51.5 & 3.663 \\
5 & -48.1 & 3.467 \\
\hline \hline
\end{tabular}

atoms are up to $\approx 1.4 \AA$ further away from the substrate as compared to the pristine nanoisland in order to accommodate the Xe.

Based on DFT calculations for the similar case of intercalated Xe penetrating the edge of an adsorbed $\mathrm{Gr}$ island [42], we assume that two edge bonds must be broken temporarily for the release of one Xe atom, requiring about $2 \mathrm{eV}$ per bond, or in total an activation energy of about $4 \mathrm{eV}$. This estimate makes plausible why $\mathrm{Xe}$ can be retained under h-BN blisters up to the highest annealing temperature of $1550 \mathrm{~K}$.

Intercalating up to $5 \mathrm{Xe}$ atoms under the 97 -atom h-BN island hardly changes the overall picture, with the adsorption energy becoming only somewhat less negative (compare Table I). When we overfill the h-BN nanoflake with 10 intercalated Xe atoms, Ir atoms are ripped out from the surface layer by the h-BN edge atoms as shown in Fig. 6(c), implying that the h-BN blister cannot anymore confine the intercalated Xe properly. This effect becomes stronger for 13 intercalated Xe atoms where the edge B atoms start to detach from $\operatorname{Ir}(111)$, enabling the escape of $\mathrm{Xe}$ atoms (for more information, see Fig. S3 of the SM).

In conclusion, our calculations for a small h-BN nanoisland with different Xe fillings indicate a very high stability of h-BN blisters and are consistent with highly pressurized Xe, unable to escape even at the highest annealing temperatures.

\section{DISCUSSION}

As outlined in the introduction, upon electron irradiation of free-standing h-BN and Gr, the two materials display a very different damage evolution. When comparing our results for h-BN on $\operatorname{Ir}(111)$ to those obtained under the same conditions for $\mathrm{Gr}$ on $\operatorname{Ir}(111)$ (compare Refs. [42,47] and related $\mathrm{SM}$ ), we find the damage evolution to be in several aspects quite similar: (i) Both materials are destroyed by room temperature ion irradiation. Upon annealing they (ii) re-establish their lattice and moiré to perfection, (iii) form highly pressurized blisters that ripen, display a transition from $2 \mathrm{D}$ to $3 \mathrm{D}$ blisters and a preferred blister attachment to 2D-layer edges, and (iv) develop a nanomesh with trapping of vacancies at specific locations of the moiré that is (v) eventually lost by the formation of large vacancy islands. We attribute this fundamental similarity to the strong influence of the substrate on the damage formation and annealing. Both materials form strong bonds between the 2D-layer edges and the substrate, which is the basis (1) to retain and reincorporate detached atoms of $\mathrm{N}, \mathrm{B}$, and $\mathrm{C}$ into the 2D layer, (2) to develop a nanomesh through the inhomogeneity of this bonding within 
the moiré, and (3) to keep the implanted Xe under the 2D layer by preventing out-diffusion at the 2D-layer edges.

As differences in the annealing behavior of h-BN and Gr we highlight the following features:

(I) For h-BN there is only one preferential location in each moiré unit cell, where vacancy clusters are pinned, namely in the area where $\mathrm{N}$ atoms would be located atop of Ir atoms. In contrast, for Gr there are two locations, the hcp and the fcc areas [47].

(II) For h-BN, the ordered positioning of the vacancy clusters is not yet fully accomplished at $1000 \mathrm{~K}$, while detachment of vacancy clusters from the moiré has already partly taken place at $1300 \mathrm{~K}$. The optimum annealing temperature to prepare a nanomesh in h-BN must be somewhere inside of this temperature interval; future work will be directed to analyze where, in order to optimize the h-BN nanomesh. For $\mathrm{Gr}$, the ordered positioning of vacancy clusters is optimal in the temperature range from 850 to $950 \mathrm{~K}$ [47], and thus at considerably lower temperatures. Why the temperature for $\mathrm{h}-\mathrm{BN}$ is higher is an open question. Since the dissolution of vacancy clusters is most likely through emission of vacancies, we speculate that the higher vacancy cluster stability may reflect a higher migration barrier of vacancy species for $h-B N$, as migration in h-BN involves homoelemental bond formation. For future work, the single pinning location and the high thermal stability of the vacancy clusters in h-BN make the determination of the optimum parameters for creation of an ordered nanomesh without large vacancy islands a very attractive topic. A removal from the substrate, e.g., through the hydrogen bubbling method [79,80], would evolve the irradiated and annealed h-BN sheet into a membrane with a very high hole density of about $1.3 \times 10^{17} \mathrm{~m}^{-2}$ (as defined by the moiré) of narrow size distribution and sizes below $1 \mathrm{~nm}$. Such a membrane would be well suitable for water desalination or gas filtration $[11,81,82]$. Even on the substrate, it would be worthwhile to explore the templating effect of an ordered array of vacancy clusters in h-BN on $\operatorname{Ir}(111)$, i.e., of the periodic array of tiny patches of bare Ir in the h-BN sheet.

(III) For h-BN on $\operatorname{Ir}(111)$, we observed at around $1500 \mathrm{~K}$ annealing temperature the thermal decomposition, while for Gr on $\operatorname{Ir}(111)$ this is not the case. This difference is closely linked to the inability to grow high-quality h-BN layers above $1300 \mathrm{~K}$ [24], while an excellent quality Gr layer can be grown at $1500 \mathrm{~K}$ on $\operatorname{Ir}(111)$ [83]. The limiting factor for the stability of h-BN is the onset of $\mathrm{B}$ and $\mathrm{N}$ species detachment from the sheet and adatom gas formation at high temperatures. Once present, atomic $\mathrm{N}$ on $\operatorname{Ir}(111)$ readily recombines to $\mathrm{N}_{2}$ which desorbs immediately at the temperatures under concern [67]. Thus, no quasiequilibrium between a 2D gas phase of its constituents and the h-BN layer on $\operatorname{Ir}(111)$ can be established. To the contrary, $\mathrm{C}$ monomers and small $\mathrm{C}$ clusters are extremely stably bound to $\operatorname{Ir}(111)$ without any chance to desorb [40]. Therefore, there is a temperature regime, extending beyond the stability range of h-BN, where small adsorbed carbon entities coexist with the Gr sheet. Only at higher temperatures does the onset of appreciable $\mathrm{C}$ solubility terminate the stability of Gr on $\operatorname{Ir}(111)$ [84,85]. It is noteworthy that upon h-BN decomposition the remaining B forms own 2D structures on $\operatorname{Ir}(111)$ [compare Fig. 2(a)], which may be worth future analysis. The fact that we observe standalone h-BN blisters at high temperatures is intimately linked to the thermal decomposition of h-BN on $\operatorname{Ir}(111)$. Standalone blisters, though not triangular, were also observed for $\mathrm{Gr}$ on Ni(111) by Späth et al. [44] and Larciprete et al. [45] under conditions where the $\mathrm{Gr}$ dissolves into the Ni bulk. The standalone blisters emerge due to the slower decay rate of the $2 \mathrm{D}$ layer separated by a gas cushion from the substrate. This mechanism may be of general relevance and we speculate that annealing $\mathrm{Gr} / \operatorname{Ir}(111)$ irradiated by noble gas to temperatures beyond $1500 \mathrm{~K}$, where dissolution of Gr into the Ir bulk sets in, would also allow us to observe standalone blisters in this case.

(IV) For h-BN, we were able to image the Xe nanocrystals within the blisters after annealing at 1000 and $1300 \mathrm{~K}$, while no superstructure indicating a solid Xe state could be resolved within Gr blisters. A simple order of magnitude estimate for the pressure inside a blister, neglecting strain effects, is obtained by equating the $p V$ work done upon compression with the adhesion per unit area $\gamma$ between h-BN and Ir gained. This yields $p=\gamma / h$, where $h$ is the height of the blister. Since h-BN adheres more strongly to $\operatorname{Ir}(111)$ than to $\mathrm{Gr}$ [26], the $\mathrm{Xe}$ in an h-BN blister may be under higher pressure, thus crystalline and visible for STM. Though this argument is highly speculative, it is also in line with another difference between blisters under the two materials. H-BN displays also blisters with a two-layer structure, while for $\mathrm{Gr}$ the transition from flat monolayer blisters is directly to smoothly curved ones.

(V) While for h-BN on $\operatorname{Ir}(111)$ vacancy islands and blisters display a clear triangular shape, for Gr on $\operatorname{Ir}(111)$ the corresponding shapes are less pronounced and mostly hexagonal [42]. For both materials, zigzag edges binding to $\operatorname{Ir}(111)$ are energetically preferred, but the heteroelemental composition reduces the symmetry-B-terminated $\mathrm{h}-\mathrm{BN}$ edges binding to Ir are strongly preferred-and implies the threefold symmetric shapes for h-BN, which is not a unique feature in consequence of ion erosion on $\operatorname{Ir}(111)$ but has also been observed for h-BN growth on $\operatorname{Ir}(111)$ or $\mathrm{Ni}(111)$ [26,76]. Also in TEM, electron irradiation (nonequilibrium conditions) experiments for free-standing h-BN results in triangular shapes [28,35], but the edges were - for reasons not entirely clear- $\mathrm{N}$ terminated rather than $\mathrm{B}$ terminated. In any case, the threefold symmetric morphology of blisters and vacancy islands, also highlighting just by shape different rotational domains, is a beautiful aspect of the irradiation morphology and so far unique to h-BN on a metal.

The state of the h-BN layer after $0.1 \mathrm{MLE}, 3 \mathrm{keV} \mathrm{Xe}{ }^{+}$ irradiation needs a brief additional discussion. In view of the large ion dose, the efficient energy deposition in the topmost layers, and a sputtering yield of 6.5 for $3 \mathrm{keV} \mathrm{Xe}^{+}$on bare Ir(111) estimated with SRIM [86], we must assume severe damage of the h-BN layer as well as roughening of the underlying $\operatorname{Ir}(111)$ substrate. This inference is consistent with the h-BN layer being LEED amorphous [no h-BN diffraction spots in the inset of Fig. 1(b)] and only very faint $\operatorname{Ir}(111)$ spots. To the extreme, one might assume it to have a melange of $\mathrm{B}, \mathrm{N}, \mathrm{Xe}$, and Ir atoms at the surface including the absence of an $\mathrm{sp}^{2}$ bonded network. Though the XPS $\mathrm{N} 1 s$ and $\mathrm{B}$ $1 s$ peaks are substantially broadened due to ion irradiation, it appears that the majority of $\mathrm{B}$ and $\mathrm{N}$ atoms are not shifted into a new chemical state, implying the preservation 
of an $\mathrm{sp}^{2}$ bonded network. The presence of an $\mathrm{sp}^{2}$ bonded network is also suggested by the ability of the sample to trap efficiently the impinging Xe (compare also Fig. S2 of the $\mathrm{SM}$ ) as well as $\mathrm{N}$ atoms. The apparent contradiction is resolved, when considering the STM data of Fig. 1(b), which displays a rough surface with a characteristic length scale of a few $\mathrm{nm}$ and an rms roughness of a few $\AA$. Since LEED is not only sensitive to lateral disorder, but also to vertical disorder, the absence of the h-BN diffraction spots does not imply the absence of a disordered $\mathrm{sp}^{2}$ bonded h-BN network. Though a precise characterization of the h-BN/Ir(111) system after irradiation is close to impossible, the data presented here appear to be consistent with a disordered and defective primarily $\mathrm{sp}^{2}$ bonded network, which covers atomic species of $\mathrm{Xe}, \mathrm{N}, \mathrm{B}$, and Ir. The preserved $\mathrm{sp}^{2}$ network with strong edge bonds to Ir, making it plausible that the h-BN layer is able to recover without the entire loss of nitrogen. It appears that the substrate not only stabilizes the $\mathrm{sp}^{2}$ bonded network upon room-temperature irradiation but also catalyzes diffusion processes in the network during annealing that help to repair the network and would not, or only at much higher temperature, be possible in free-standing h-BN monolayers. To the best of our knowledge, to date the only other irradiation data for h-BN on a metal substrate is by Cun et al. [49-51] complemented by the theoretical modeling of Iannuzzi [52]. Though the parameters of irradiation are very different (extremely low fluence, very low ion energy of the order of $100 \mathrm{eV}$, and $\mathrm{Ar}^{+}$ instead of $\mathrm{Xe}^{+}$), several similarities to our work can be noted: (i) Ar atoms are trapped underneath the h-BN sheet on $\mathrm{Rh}(111)$ and upon annealing they become mobile and aggregate to small blisters. (ii) Vacancies created at room temperature aggregate upon annealing and assemble at a specific location of the moiré, as for Xe irradiation of h-BN on $\operatorname{Ir}(111)$. (iii) Above around $1000 \mathrm{~K}$, the vacancy clusters unpin from the moiré and aggregate to larger islands [for h-BN on $\operatorname{Ir}(111)$ unpinning takes place above $1300 \mathrm{~K}$ ]. At variance with our findings is the disappearance of noble-gas-related protrusions already annealing to $900 \mathrm{~K}$ [49], hard to reconcile with the fact that blisters are the objects of highest thermal stability for h-BN on $\operatorname{Ir}(111)$, objects that even survive the decomposition of h-BN in direct contact with $\operatorname{Ir}(111)$. Also at variance with our results is the observation of small cut-out flakes after annealing to $900 \mathrm{~K}$. They appear as bright bumps in STM [49]. A unified view on the phenomena in h-BN on the two metals would emerge, if the bright bumps would be interpreted as blisters, rather than as cut-out flakes.

\section{CONCLUSIONS}

Our investigations show that the global damage and annealing morphology of $\mathrm{h}-\mathrm{BN}$ and $\mathrm{Gr}$ on $\operatorname{Ir}(111)$ bear strong similarities, involving irradiation induced structural disintegration, re-establishment of lattice and moiré pattern, blister and nanomesh (ordered positioning of vacancy clusters) formation, and final collapse of the vacancy clusters to large vacancy islands at the highest annealing temperature. Despite the dissimilarity of the electron irradiation damage of the free-standing materials, these similarities for the supported materials arise from the strong bonding of the 2D-layer edges to the $\operatorname{Ir}(111)$ substrate. We believe this feature to be of general relevance and expect for any 2D layer on a substrate, to which its edges bind strongly, a similar set of phenomena after irradiation and annealing, as described here. Presumably due to the stronger adhesion and thus comparatively higher pressure in h-BN blisters as compared to $\mathrm{Gr}$ ones, we were able to directly image solid $\mathrm{Xe}$ of monolayer and bilayer thickness through the blister cover. We thereby provide a direct proof for the high pressures in irradiation-induced noble gas blisters under 2D layers on a metal. At sufficiently high annealing temperatures, standalone blisters only sealed to the metal substrate form. We identified the onset of 2D-layer decomposition as the key condition for the creation of standalone blisters. Visually, an appealing effect of the heteroelemental binding in h-BN is the development of pronounced triangular shapes in the entire morphology, which is traced back to the strong and preferential binding of B-terminated zigzag edges to $\operatorname{Ir}(111)$. We found that each moiré unit cell can trap just one vacancy cluster at a specific location, namely the chemisorbed valley region of the h-BN layer. This uniqueness of vacancy cluster pinning makes h-BN on $\operatorname{Ir}(111)$ from the fundamental point of view superior to $\mathrm{Gr} / \mathrm{Ir}(111)$ and may thus enable the formation of much better ordered arrays of vacancy clusters.

\section{ACKNOWLEDGMENTS}

The authors are grateful for the financial support of the Deutsche Forschungsgemeinschaft (DFG) through Project No. MI581/22-1, Low Energy Ion Irradiation of 2D Materials, and of the Bonn-Cologne Graduate School for Physics and Astronomy (C.H.). The MAX IV Laboratory personnel are acknowledged for support during measurements. J.K. and M.A. are grateful for financial support by Project Grant No. 2012-3850 financed by the Swedish Research Council. The computations were performed at the high-performance computer JUQUEEN operated by the JSC at the Forschungszentrum Juelich. V. Caciuc and N. Atodiresei acknowledge financial support from the Volkswagen-Stiftung through the Optically Controlled Spin Logic project and by the DFG through the Collaborative Research Center SFB 1238 (Project No. C01).
[1] M. Topsakal, E. Aktürk, and S. Ciraci, Phys. Rev. B 79, 115442 (2009).

[2] H. Dil, J. Lobo-Checa, R. Laskowski, P. Blaha, S. Berner, J. Osterwalder, and T. Greber, Science 319, 1824 (2008).

[3] F. Schulz, R. Drost, S. Hämäläinen, and P. Liljeroth, ACS Nano 7, 11121 (2013).
[4] P. Gehring, B. Gao, M. Burghard, and K. Kern, Nano Lett. 12, 5137 (2012).

[5] C. Dean, A. Young, I. Meric, C. Lee, L. Wang, S. Sorgenfrei, K. Watanabe, T. Taniguchi, P. Kim, K. Shepard et al., Nat. Nanotechnol. 5, 722 (2010). 
[6] L. Britnell, R. Gorbachev, R. Jalil, B. Belle, F. Schedin, A. Mishchenko, T. Georgiou, M. Katsnelson, L. Eaves, S. Morozov et al., Science 335, 947 (2012).

[7] F. Withers, O. Del Pozo-Zamudio, A. Mishchenko, A. Rooney, A. Gholinia, K. Watanabe, T. Taniguchi, S. Haigh, A. Geim, A. Tartakovskii et al., Nat. Mater. 14, 301 (2015).

[8] K. Gopinadhan, Y. Shin, R. Jalil, T. Venkatesan, A. Geim, A. Neto, and H. Yang, Nat. Commun. 6, 8337 (2015).

[9] D. Golberg, Y. Bando, Y. Huang, T. Terao, M. Mitome, C. Tang, and C. Zhi, ACS Nano 4, 2979 (2010).

[10] W. Lei, D. Portehault, D. Liu, S. Qin, and Y. Chen, Nat. Commun. 4, 1777 (2013).

[11] J. Azamat, A. Khataee, and F. Sadikoglu, RSC Adv. 6, 94911 (2016).

[12] M. Yamaguchi, J. Bernhardt, K. Faerstein, D. Shtansky, Y. Bando, I. Golovin, H. Sinning, and D. Golberg, Acta Mater. 61, 7604 (2013).

[13] A. Pakdel, Y. Bando, and D. Golberg, ACS Nano 8, 10631 (2014).

[14] M. Corso, W. Auwärter, M. Muntwiler, A. Tamai, T. Greber, and J. Osterwalder, Science 303, 217 (2004).

[15] R. Laskowski and P. Blaha, J. Phys.: Condens. Matter 20, 064207 (2008).

[16] S. Berner, M. Corso, R. Widmer, O. Groening, R. Laskowski, P. Blaha, K. Schwarz, A. Goriachko, H. Over, S. Gsell et al., Angew. Chem., Int. Ed. 46, 5115 (2007).

[17] A. Goriachko, Y. He, M. Knapp, H. Over, M. Corso, T. Brugger, S. Berner, J. Osterwalder, and T. Greber, Langmuir 23, 2928 (2007).

[18] P. Sutter, J. Lahiri, P. Albrecht, and E. Sutter, ACS Nano 5, 7303 (2011).

[19] J. Lu, P. Yeo, Y. Zheng, H. Xu, C. Gan, M. Sullivan, A. Castro Neto, and K. Loh, J. Am. Chem. Soc. 135, 2368 (2013).

[20] E. Ćavar, R. Westerström, A. Mikkelsen, E. Lundgren, A. Vinogradov, M. L. Ng, A. Preobrajenski, A. Zakharov, and N. Mårtensson, Surf. Sci. 602, 1722 (2008).

[21] F. Orlando, R. Larciprete, P. Lacovig, I. Boscarato, A. Baraldi, and S. Lizzit, J. Phys. Chem. C 116, 157 (2011).

[22] F. Orlando, P. Lacovig, L. Omiciuolo, N. Apostol, R. Larciprete, A. Baraldi, and S. Lizzit, ACS Nano 8, 12063 (2014).

[23] A. Preobrajenski, M. Nesterov, M. L. Ng, A. Vinogradov, and N. Mårtensson, Chem. Phys. Lett. 446, 119 (2007).

[24] D. Usachov, A. Fedorov, O. Vilkov, V. K. Adamchuk, L. V. Yashina, L. Bondarenko, A. A Saranin, A. Grüneis, and D. V. Vyalikh, Phys. Rev. B 86, 155151 (2012).

[25] F. Schulz, R. Drost, S. K. Hämäläinen, T. Demonchaux, A. P. Seitsonen, and P. Liljeroth, Phys. Rev. B 89, 235429 (2014).

[26] F. Farwick zum Hagen, D. Zimmermann, C. Silva, C. Schlueter, N. Atodiresei, W. Jolie, A. Martínez-Galera, D. Dombrowski, U. Schröder, M. Will et al., ACS Nano 10, 11012 (2016).

[27] M. Petrović, U. Hagemann, M. Horn-von Hoegen, and F.-J. M. zu Heringdorf, Appl. Surf. Sci. 420, 504 (2017).

[28] J. Meyer, A. Chuvilin, G. Algara-Siller, J. Biskupek, and U. Kaiser, Nano Lett. 9, 2683 (2009).

[29] J. Kotakoski, C. H. Jin, O. Lehtinen, K. Suenaga, and A. V. Krasheninnikov, Phys. Rev. B 82, 113404 (2010).

[30] J. Warner, E. Margine, M. Mukai, A. Robertson, F. Giustino, and A. Kirkland, Science 337, 209 (2012).

[31] O. Lehtinen, S. Kurasch, A. V. Krasheninnikov, and U. Kaiser, Nat. Commun. 4, 2098 (2013).
[32] A. Robertson, G. Lee, K. He, E. Yoon, A. Kirkland, and J. Warner, Nano Lett. 14, 1634 (2014).

[33] J. C. Meyer, C. Kisielowski, R. Erni, M. D. Rossell, M. Crommie, and A. Zettl, Nano Lett. 8, 3582 (2008).

[34] J. Kotakoski, A. V. Krasheninnikov, U. Kaiser, and J. C. Meyer, Phys. Rev. Lett. 106, 105505 (2011).

[35] C. Jin, F. Lin, K. Suenaga, and S. Iijima, Phys. Rev. Lett. 102, 195505 (2009).

[36] A. Krasheninnikov, P. Lehtinen, A. Foster, and R. Nieminen, Chem. Phys. Lett. 418, 132 (2006).

[37] A. Zobelli, C. P. Ewels, A. Gloter, and G. Seifert, Phys. Rev. B 75, 094104 (2007).

[38] H. Cun, M. Iannuzzi, A. Hemmi, J. Osterwalder, and T. Greber, Surf. Sci. 634, 95 (2015).

[39] H. Kim, W. Ko, J. Ku, I. Jeon, D. Kim, H. Kwon, Y. Oh, S. Ryu, Y. Kuk, S. Hwang et al., Nat. Commun. 6, 7528 (2015).

[40] C. Herbig, E. Åhlgren, W. Jolie, C. Busse, J. Kotakoski, A. Krasheninnikov, and T. Michely, ACS Nano 8, 12208 (2014).

[41] C. Herbig, E. Åhlgren, U. Schröder, A. Martínez-Galera, M. Arman, W. Jolie, C. Busse, J. Kotakoski, J. Knudsen, A. Krasheninnikov et al., ACS Nano 9, 4664 (2015).

[42] C. Herbig, E. H. Åhlgren, U. A. Schröder, A. J. Martínez-Galera, M. A. Arman, J. Kotakoski, J. Knudsen, A. V. Krasheninnikov, and T. Michely, Phys. Rev. B 92, 085429 (2015).

[43] G. Zamborlini, M. Imam, L. L. Patera, T. O. Mentes, N. Stojić, C. Africh, A. Sala, N. Binggeli, G. Comelli, and A. Locatelli, Nano Lett. 15, 6162 (2015).

[44] F. Späth, K. Gotterbarm, M. Amende, U. Bauer, C. Gleichweit, O. Höfert, H. Steinrück, and C. Papp, Surf. Sci. 643, 222 (2016).

[45] R. Larciprete, S. Colonna, F. Ronci, R. Flammini, P. Lacovig, N. Apostol, A. Politano, P. Feulner, D. Menzel, and S. Lizzit, Nano Lett. 16, 1808 (2016).

[46] E. H. Åhlgren, S. K. Hämäläinen, O. Lehtinen, P. Liljeroth, and J. Kotakoski, Phys. Rev. B 88, 155419 (2013).

[47] S. Standop, O. Lehtinen, C. Herbig, G. Lewes-Malandrakis, F. Craes, J. Kotakoski, T. Michely, A. V. Krasheninnikov, and C. Busse, Nano Lett. 13, 1948 (2013).

[48] C. Herbig and T. Michely, 2D Mater 3, 025032 (2016).

[49] H. Cun, M. Iannuzzi, A. Hemmi, S. Roth, J. Osterwalder, and T. Greber, Nano Lett. 13, 2098 (2013).

[50] H. Cun, M. Iannuzzi, A. Hemmi, J. Osterwalder, and T. Greber, ACS Nano 8, 7423 (2014).

[51] H. Cun, M. Iannuzzi, A. Hemmi, J. Osterwalder, and T. Greber, ACS Nano 8, 1014 (2013).

[52] M. Iannuzzi, J. Phys. Chem. C 119, 22198 (2015).

[53] See Supplemental Material at http://link.aps.org/supplemental/ 10.1103/PhysRevB.96.235410 contains a STM irradiation and annealing sequence for $0.5 \mathrm{keV} \mathrm{Xe}{ }^{+}$irradiation of h-BN on $\operatorname{Ir}(111)$ (Fig. S1), the results of XPS experiments documenting the trapping of Xe underneath the h-BN layer (Fig. S2), additional DFT calculations for the Xe-filled blisters with different amounts of Xe intercalated beneath the h-BN nanoflake (Fig. S3), and the details of the fit components used in Fig. 5 (Table S1).

[54] R. Nyholm, J. Andersen, U. Johansson, B. Jensen, and I. Lindau, Nucl. Instrum. Methods Phys. Res., Sect. A 467, 520 (2001).

[55] P. Hohenberg and W. Kohn, Phys. Rev. 136, B864 (1964).

[56] P. E. Blöchl, Phys. Rev. B 50, 17953 (1994).

[57] G. Kresse and J. Hafner, Phys. Rev. B 47, 558 (1993).

[58] G. Kresse and J. Furthmüller, Phys. Rev. B 54, 11169 (1996). 
[59] G. Kresse and D. Joubert, Phys. Rev. B 59, 1758 (1999).

[60] J. P. Perdew, K. Burke, and M. Ernzerhof, Phys. Rev. Lett. 77, 3865 (1996).

[61] K. Lee, E. D. Murray, L. Kong, B. I. Lundqvist, and D. C. Langreth, Phys. Rev. B 82, 081101(R) (2010).

[62] I. Hamada, Phys. Rev. B 89, 121103(R) (2014).

[63] S. Joshi, D. Ecija, R. Koitz, M. Iannuzzi, A. Seitsonen, J. Hutter, H. Sachdev, S. Vijayaraghavan, F. Bischoff, and K. Seufert, Nano Lett. 12, 5821 (2012).

[64] O. Lehtinen, E. Dumur, J. Kotakoski, A. Krasheninnikov, K. Nordlund, and J. Keinonen, Nucl. Instr. Meth. Phys. Res. B 269, 1327 (2011).

[65] C. Herbig, E. H. Åhlgren, and T. Michely, Nanotechnology 28, 055304 (2016).

[66] B. Feng, J. Zhang, Q. Zhong, W. Li, S. Li, H. Li, P. Cheng, S. Meng, L. Chen, and K. Wu, Nat. Chem. 8, 563 (2016).

[67] A. Santra, B. Min, C. Yi, K. Luo, T. Choudhary, and D. Goodman, J. Phys. Chem. B 106, 340 (2002).

[68] J. C. L. Cornish and N. R. Avery, Surf. Sci. 235, 209 (1990).

[69] P. Mallet, F. Varchon, C. Naud, L. Magaud, C. Berger, and J. Y. Veuillen, Phys. Rev. B 76, 041403 (2007).

[70] A. Martínez-Galera, I. Brihuega, and J. Gómez-Rodríguez, Nano Lett. 11, 3576 (2011).

[71] A. Martínez-Galera, U. Schröder, F. Huttmann, W. Jolie, F. Craes, C. Busse, V. Caciuc, N. Atodiresei, S. Blügel, and T. Michely, Nanoscale 8, 1932 (2016).

[72] S. Schumacher, F. Huttmann, M. Petrović, C. Witt, D. Förster, C. Vo-Van, J. Coraux, A. J. Martínez-Galera, V. Sessi, I. Vergara et al., Phys. Rev. B 90, 235437 (2014).
[73] P. R. Granfors, A. T. Macrander, and R. O. Simmons, Phys. Rev. B 24, 4753 (1981).

[74] K. Schubert, Acta Crystallogr. Sect. B 30, 193 (1974).

[75] D. A. Young, Phase Diagrams of the Elements (University of California Press, Berkeley, 1991).

[76] W. Auwärter, M. Muntwiler, J. Osterwalder, and T. Greber, Surf. Sci. 545, L735 (2003).

[77] J. Knudsen, P. J. Feibelman, T. Gerber, E. Grånäs, K. Schulte, P. Stratmann, J. N. Andersen, and T. Michely, Phys. Rev. B 85, 035407 (2012).

[78] K. Ueda, K. Suzuki, R. Toyoshima, Y. Monya, M. Yoshida, K. Isegawa, K. Amemiya, K. Mase, B. Mun, M. Arman et al., Top. Catal. 59, 487 (2016).

[79] E. Koren, E. Sutter, S. Bliznakov, F. Ivars-Barcelo, and P. Sutter, Appl. Phys. Lett. 103, 121602 (2013).

[80] I. Rakić, D. Čapeta, M. Plodinec, and M. Kralj, Carbon 96, 243 (2016).

[81] D. Cohen-Tanugi and J. Grossman, Nano Lett. 12, 3602 (2012).

[82] D. Cohen-Tanugi, L. Lin, and J. Grossman, Nano Lett. 16, 1027 (2016).

[83] H. Hattab, A. N'Diaye, D. Wall, G. Jnawali, J. Coraux, C. Busse, R. Van Gastel, B. Poelsema, T. Michely, F. Meyer zu Heringdorf et al., Appl. Phys. Lett. 98, 141903 (2011).

[84] S. Nie, A. Walter, N. Bartelt, E. Starodub, A. Bostwick, E. Rotenberg, and K. McCarty, ACS Nano 5, 2298 (2011).

[85] E. Loginova, N. Bartelt, P. Feibelman, and K. McCarty, New J. Phys. 11, 063046 (2009).

[86] J. Ziegler, M. Ziegler, and J. Biersack, Nucl. Instrum. Methods Phys. Res., Sect. B 268, 1818 (2010). 